Larissa M. Bieler / Maren Runte

\title{
Semantik der Sinne
}

\section{Die lexikografische Erfassung von Geschmacksadjektiven ${ }^{1}$}

\author{
$1 \quad$ Einleitung \\ 2 Zur Lemmaselektion: \\ Der Geschmackswortschatz \\ des Deutschen \\ 3 Zur Datenerhebung mit Fokusgruppen \\ 3.1 Die Fokusgruppendiskussion \\ als linguistisches Instrument \\ 3.1.1 Überlegungen zur Methodenwahl
}

\author{
3.1.2 Die Fokusgruppe als conceptual society \\ 3.1.3 Methodenfazit \\ 4 Strategien zur Versprachlichung \\ von Geschmackswahrnehmungen \\ 4.1 Strategien auf paradigmatischer Ebene \\ 4.2 Anbindung an die Lebenswelt \\ $5 \quad$ Fazit \\ 6 Literatur
}

\section{Abstract}

This contribution aims at presenting an approach towards an adequate presentation of German taste terms in a lexicographic data base. The lexicographic project builds up on existing data, which were collected in the context of the research project "Sensory Language and the Semantics of Taste" and which contain discussions about taste perception. These focus-group discussions are transcribed and examined to find strategies of how taste perception is described in everyday life. In this paper we would like to present these strategies as well as their integration in the data base.

De gustibus non est disputandum. Jean Anthèlme Brillat-Savarin

\section{$1 \quad$ Einleitung}

Über Geschmack lässt sich bekanntlich nicht streiten. Tatsächlich sind im Alltag Streitgespräche über die gustatorische Wahrnehmung von Lebensmitteln und Gerichten - was in der Alltagssprache im Gegensatz zur Fachsprache der Lebensmittelsensorik als „Geschmack“ bezeichnet wird - eher selten. Eine wesentliche Voraussetzung für derartige Diskussionen bestünde in einer klar umgrenzten und genau definierten Terminologie zur Beschreibung der Sinneseindrücke, wie sie etwa in bestimmten Fachsprachen im gastronomischen Bereich (z.B. der Weinsprache) oder in der Lebensmittelsensorik vorliegt.

Dass für die Beschreibung von Geschmackswahrnehmungen im Alltag kein Wortschatz mit diesen Merkmalen zur Verfügung steht, liegt einerseits daran, dass die Wahrnehmung von Geschmack - physiologisch betrachtet - individuell ist. Gründe hierfür sind neben individuellen Wahrnehmungsschwellen für bestimmte Geschmacksqualitäten insbesondere

1 Für Diskussionen im Vorfeld der Entstehung des vorliegenden Beitrags sowie für hilfreiche kritische Anmerkungen zu Vorfassungen des Textes danken wir Jeannette Nuessli Guth und insbesondere Angelika Linke herzlich. 
individuelle Erfahrungswerte, auf deren Grundlage neue Geschmackswahrnehmungen beurteilt werden (vgl. Bierbaumer/Schmidt 2006:441). Andererseits hängt auch die Fähigkeit, Sinneseindrücke in Worte zu fassen, von den sprachlichen Fähigkeiten des Einzelnen ab; Beschreibungen von Geschmack sind daher notwendigerweise subjektiv.

Hinzu kommt, dass Geschmackswahrnehmung und deren Verbalisierung historisch, kulturell und durch soziale Faktoren geprägt sind. An dieser Stelle mag der unterschiedliche Schärfegrad (und dessen Beurteilung) von Gerichten verschiedener Nationalküchen oder aber die unterschiedliche Kenntnis von bzw. der unterschiedliche Umgang mit Gewürzen und Lebensmitteln als Illustration genügen.

Unter lexikalischen Gesichtspunkten sind die meisten Wörter, mit denen im Alltag Geschmackswahrnehmungen beschrieben werden, polysem und werden häufig metaphorisch verwendet (etwa einen bitteren Geschmack hinterlassen, ein süßes Mädchen, sauer aufstoßen, s. auch Wagner 2009:139f.). Aber auch bei der konkreten Verwendung in Zusammenhang mit Geschmacksbeschreibungen zeichnen sich diese Wörter häufig durch einen hohen Grad an Vagheit und Kontextabhängigkeit aus (s. auch Bieler et al., im Druck). Was genau hat man sich etwa unter dem angepriesenen frischen Fisch vorzustellen? Einen frisch gefangenen, einen frisch zubereiteten oder etwa einen frisch aufgetauten Fisch?

Tatsächlich scheint die Beschreibung von Geschmack in Alltagsgesprächen aber nicht problematisch zu sein. Bei näherer Beschäftigung mit der Thematik zeigt sich, dass vielfältige Strategien zur Verfügung stehen, um die individuelle Geschmackswahrnehmung interpersonell zu vermitteln. Hierbei reichen die Strategien von der Beschreibung von Geschmackswahrnehmung mit Hilfe einzelner Geschmackswörter über die Verwendung von Kontrastierungen und den Einsatz von Gestik bis hin zu ganzen narrativen Sequenzen (vgl. Abschnitt 4).

Das interdisziplinäre Forschungsprojekt Sensory Language and the Semantics of Taste (SenS), das den Forschungshintergrund zum vorliegenden Beitrag bildet ${ }^{2}$, hat sich zum Ziel gesetzt, den Geschmackswortschatz des Deutschen zu erfassen, die Verbalisierung von Geschmacksempfindungen zu untersuchen und Geschmackswortschatz wie auch Verbalisierungsstrategien - basierend auf dem uns vorliegenden Datenmaterial - darzustellen. Langfristiges Ziel des Projekts soll es sein, unsere Arbeitsergebnisse in Form einer lexikografischen Datenbank zu erfassen.

Mögliche Adressaten unserer Untersuchungsergebnisse sind Personen, die mehr über die Bedeutung und Verwendung von Geschmackswörtern im Alltag erfahren möchten als allgemein einsprachige Wörterbücher der deutschen Sprache enthalten. Die Konzeption zielt daher im philologischen Bereich auf LinguistInnen, im nicht-philologischen Bereich auf Wissenschaftler, die sich näher mit der Beschreibung von Geschmackswahrnehmungen beschäftigen (also LebensmittelsensorikerInnen, ErnährungswissenschaftlerInnen und Fachleute aus der Lebensmittelindustrie), und im staatlichen Bereich auf Personen, die sich mit der Zulassung und Qualitätskontrolle von Lebensmitteln befassen. Im Bereich Marketing könnten unsere Ergebnisse auch für diejenigen interessant sein, die den Geschmack von Produkten adäquat beschreiben müssen.

2 An diesem Projekt sind Forschende der Universitäten Basel und Zürich, der ETH Zürich sowie der Zürcher Hochschule für angewandte Wissenschaften ZHAW beteiligt. Weitere Informationen sind auf der Projektseite www.sensorysemantics.ch zu finden. 
Im Folgenden möchten wir die Erhebungsmethoden und bereits vorliegende Untersuchungsergebnisse näher vorstellen, wobei wir uns insbesondere auf die Methode der Fokusgruppendiskussion sowie auf die Darstellung der in den Fokusgruppengesprächen verwendeten Strategien zur Verbalisierung von Geschmackswahrnehmungen konzentrieren möchten. Abschließend soll deren Einbindung in die geplante lexikografische Datenbank thematisiert werden.

\section{Zur Lemmaselektion: Der Geschmackswortschatz des Deutschen}

Geschmack schließt chemisch-biologisch betrachtet alles das ein, was bei der Aufnahme von Nahrung im Mund- und Rachenraum wahrgenommen wird. In der Terminologie der Lebensmittelsensorik ist „Geschmack“ dagegen auf die Geschmackswahrnehmungen im Mundraum und daher auf die Wahrnehmung der Grundgeschmacksqualitäten beschränkt (vgl. Ternes et al., 2005:684). Diese Reize werden auf der Zunge mit Hilfe von spezifischen Rezeptoren in den so genannten „Geschmacksknospen“ wahrgenommen.

Der eigentliche Geschmackswortschatz des Deutschen ist somit sehr klein und umfasst ursprünglich allein die vier Grundgeschmacksqualitäten süß, sauer, bitter und salzig. Anfang des 20. Jahrhunderts ist das Modell der vier Grundgeschmacksqualitäten um eine weitere - heute in der Lebensmittelsensorik allgemein anerkannte - Geschmacksqualität erweitert worden: umami. Der Geschmack umami basiert auf Natriumglutamat als Inhaltsstoff; typische Lebensmittel, die umami schmecken, sind Bouillon, Tomatenkonzentrat und Parmesankäse, weshalb die Geschmacksempfindung im Alltag u.a. mit bouillonartig oder würzig umschrieben wird. Der Terminus umami hat sich zwar inzwischen in der lebensmittelsensorischen Fachsprache durchgesetzt, in der Alltagssprache wird das Wort aber nur selten verwendet bzw. ist gänzlich unbekannt ${ }^{3}$.

Bereits bei den Grundgeschmacksqualitäten zeigt sich der Einfluss des jeweiligen Kulturkreises auf die Geschmackswahrnehmung und -beschreibung. Im Deutschen besteht durch das Fehlen eines äquivalenten, lexikalisierten Ausdrucks für umami eine lexikalische Lücke, die in anderen Kulturen - etwa der Japans oder Indonesiens - durchaus besetzt ist (vgl. auch Wagner 2009:136).

Im Alltag lässt sich die Beschreibung von Geschmackswahrnehmungen allerdings nicht nur auf vier Wörter - süß, sauer, bitter und salzig - reduzieren. Das hat zum einen physiologische Gründe: Beim Kauen von Nahrung werden in der Mundhöhle Aromastoffe freigesetzt, welche in der Nase wahrgenommen werden. Daher lassen sich Geschmack und Geruch nicht leicht voneinander trennen, der Gesamteindruck der Wahrnehmung ist entscheidend. Viele Wahrnehmungen beim Verzehr von Nahrung sind Aromawahrnehmungen, die in der Sprache oft als Geschmack beschrieben werden.

3 Im Duden-Universalwörterbuch wird zwar eine knappe, allerdings wenig hilfreiche Definition von umami angegeben (,in einer Geschmacksrichtung liegend, die weder süß noch sauer, bitter od. salzig ist", DUW 2007), im Wahrig ist umami dagegen nicht in der Lemmaliste enthalten. Auch in der Alltagssprache wird umami kaum verwendet: Von den 226 Teilnehmenden des „Basic Taste Term Tests“ (vgl. Abschnitt 2) haben nur zwei Personen das Geschmackswort genannt. 
Zum anderen machen wir bei der Beschreibung von Geschmack im Alltag mehr als nur eine Aussage über die Qualität des Geschmacks: Wir sagen etwas über die Intensität des Geschmacks (geschmacklos, mild, scharf etc.), über den Wohlgeschmack eines Lebensmittels (gut, schlecht, lecker etc.) und sogar über die Konsistenz bzw. Textur der Produkte (cremig, knusprig, knackig etc.) aus.

Auch diese Aussagen sind für die Verbalisierung von Geschmack im Alltag wesentlich; Wörter, welche einem dieser vier Bereiche zugeordnet werden können, müssen daher auch als Geschmackswörter gewertet werden.

Die Erfassung des tatsächlichen, im Alltag verwendeten Geschmackswortschatzes des Deutschen basiert im Rahmen des Projekts auf drei Erhebungsverfahren:

1. Der Erhebung von Geschmackswörtern in verschiedenen allgemeinen einsprachigen Wörterbüchern des Deutschen ${ }^{4}$ :

Die Wörterbücher wurden daraufhin überprüft, welche Lexeme für die Beschreibung von Geschmack verwendet werden. Entscheidend bei der Durchsicht der Wörterbücher war, dass die Wörter in mindestens einer Lesart in Zusammenhang mit Geschmack bzw. Aroma oder schmecken verwendet werden; diese Lexeme mussten explizit innerhalb der lexikografischen Bedeutungsbeschreibung bzw. bei den Beispielangaben genannt werden.

Anschließend wurden die Wörter in einer Wortliste nach ihrer Wortart sortiert, wobei wir uns im Anschluss nur auf die zahlreichen Geschmacksadjektive konzentriert haben.

2. Der Auswertung des größten schriftsprachlichen Korpus der deutschen Sprache:

Auch das größte Korpus des Instituts für deutsche Sprache (IDS) in Mannheim, COSMAS II, wurde mit identischen Kriterien auf Geschmackswörter durchsucht. Die Liste der in den Wörterbüchern verzeichneten Geschmacksadjektive konnte daraufhin überprüft, ergänzt und erweitert werden.

Auf diesem Weg sollte einerseits der tatsächliche Sprachgebrauch abgebildet werden, und andererseits sollten auch Adjektive zur Geschmacksbeschreibung erfasst werden, die in den Wörterbüchern nicht in Kontext mit Geschmackswahrnehmungen verzeichnet werden.

Da die Liste der so erhobenen Geschmacksadjektive sehr umfangreich war (ca. 600 Lemmata), blieb zunächst die Frage offen, mit Hilfe welcher Methode man zwischen Zentrum und Peripherie des Geschmackswortschatzes unterscheiden kann. Ist etwa das Adjektiv reich als Geschmackswort zu werten, weil sich in COSMAS - wenn auch nur wenige - Beispiele für eine Verwendung im Geschmackskontext finden lassen? ${ }^{5}$

3. Der Durchführung des sog. „Basic Taste Term Tests“ an verschiedenen Schweizer Hochschulen 6 :

4 Untersucht wurden das Deutsche Universalwörterbuch aus dem Duden-Verlag (DUW) von 2007, das Deutsche Wörterbuch von Wahrig von 2006, Der deutsche Wortschatz nach Sachgruppen von Dornseiff in der Auflage von 2004, das Variantenwörterbuch des Deutschen von 2004 und das Deutsche Wörterbuch von Hermann Paul in der 10. Auflage von 2006.

5 Ein Beispiel für die seltene Verwendung von reich im Zusammenhang mit Geschmack/Aroma lässt sich in der Kleinen Zeitung vom 14.03.2000 finden: „Ein reiches Aroma wird dem Sonnenblumenöl attestiert, welches häufig über die Ladentische wandert.“

6 Der Test wurde durchgeführt an der Zürcher Hochschule für Angewandte Wissenschaften (Departement Angewandte Linguistik), der Universität Zürich (Deutsches Seminar) und der Universität Basel (Deutsches Seminar). 
In Anlehnung an das Vorgehen von Morgan und Corbett (vgl. Morgan/Corbett 1989), die diesen Test im Rahmen ihrer Forschung zum Grundwortschatz der Farben durchgeführt haben, wurden 226 Testteilnehmer gebeten, innerhalb eines begrenzten Zeitraums möglichst viele Wörter aufzuschreiben, mit denen der Geschmack von Lebensmitteln beschrieben werden kann. Im Testverfahren wurden auch die Einträge pro Minute registriert, so dass bei der Auswertung sowohl die Zahl der Nennungen als auch der Zeitpunkt der Nennung eine entscheidende Rolle spielten.

Auf diese Weise konnten weitere Geschmacksadjektive, aber auch der zentrale Geschmackswortschatz des Deutschen ermittelt werden: Es sind einerseits die Lexeme, die am häufigsten genannt werden, andererseits aber auch die Lexeme, die besonders häufig in der ersten Minute genannt werden.

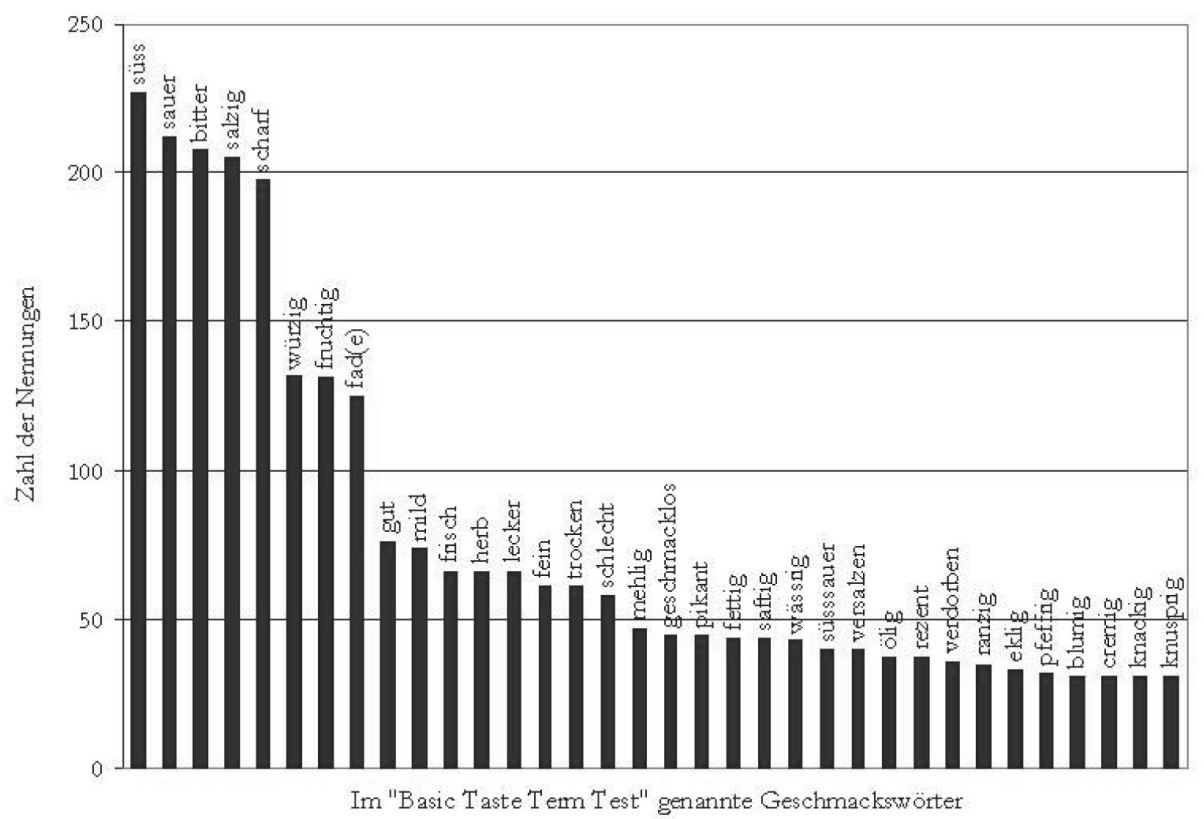

Diagramm 1: Ergebnisse des „Basic Taste Term Tests“ (insgesamt)

Das Auswertungsergebnis demonstriert, wie dominant die vier Grundgeschmackswörter unter den am meisten genannten Geschmackswörtern sind. Dass hierunter der Terminus umami fehlt, zeigt, dass sich das Lexem (noch) nicht in der Alltagssprache durchgesetzt hat.

Interessant ist ebenfalls, dass von den Sprechern scharf ebenso häufig und schnell genannt wird wie die Grundgeschmacksqualitäten und daher auch zum zentralen Geschmackswortschatz des Deutschen zu zählen ist.

Weiterhin wird deutlich, welche wesentliche Rolle hedonische Geschmacksadjektive (gut, lecker, schlecht, fein) sowie Adjektive, die eine Geschmacksintensität bezeichnen (würzig, fad, mild, pikant), spielen. 
Ausgehend von den Ergebnissen dieser Auswertungen wird im Rahmen von SenS eine Beschreibung der wichtigsten Geschmacksadjektive der deutschen Sprache erarbeitet.

\section{Zur Datenerhebung mit Fokusgruppen}

Einerseits ist für die Bedeutungsbeschreibung der Geschmacksadjektive die Erhebung und Auswertung schriftlicher Belege unverzichtbar. Im Rahmen von SenS nutzen wir daher COSMAS II, wobei wir uns bei den Suchabfragen auf solche Belege beschränken, in denen die Lexeme im Zusammenhang mit Geschmack verwendet werden; die metaphorische Verwendung wird dabei nicht berücksichtigt. Die schriftsprachlichen Belege dienen anschließend u.a. dazu, typische Verwendungskontexte des jeweiligen Geschmackswortes wie auch häufige genannte Referenzen zu eruieren.

Andererseits ist aufgrund der Individualität von Geschmackswahrnehmungen auch die Erhebung und Untersuchung von mündlichen Sprachdaten äußerst interessant, um zu erforschen, auf welche Weise Geschmackswahrnehmungen interpersonell vermittelt werden. Daher soll im Folgenden die im Rahmen von SenS angewandte Methode der Fokusgruppendiskussion näher beschrieben werden.

\subsection{Die Fokusgruppendiskussion als linguistisches Instrument}

Fokusgruppen sind zeitlich begrenzte Gruppendiskussionen mit einer begrenzten Teilnehmerzahl. Die Thematik der Gruppendiskussion ist durch das Erkenntnisinteresse der Forschenden bestimmt. Die Moderation beschränkt sich dabei auf die Aktivierung des Gesprächs, damit sich ein freier und ,selbstläufiger Diskurs“ (Schäffer 2003:76) entwickelt. In Fokusgruppen kann eine relativ entspannte und lockere Gesprächsatmosphäre geschaffen werden, die es den Teilnehmenden erleichtert, sich in einer ,gewohnten Sprachform und unter Rückgriff auf vertraute Relevanzsysteme zu äußern“ (Abel/Stuflesser 2009:80).

Im Rahmen des Projekts wurden bislang 15 Fokusgruppen zu Geschmackswörtern (bspw. knackig, fettig) sowie 15 Gruppen zu Produkten (bspw. Brot, Joghurt) durchgeführt, wobei an diesen Gesprächen jeweils zwischen sechs und acht Personen beteiligt waren. Die Fokusgruppen wurden mehrheitlich mit Sprecherinnen und Sprechern im Alter zwischen 20 und 35 Jahren besetzt. Zu den meisten Begriffen oder Produkten wurden je eine Männer- und eine Frauengruppe durchgeführt, wobei wir uns aufgrund der Zielsetzung, den standardsprachlichen Geschmackswortschatz der deutschen Sprache zu erforschen, auf Erhebungen mit Standardsprecherinnen und -sprechern konzentriert haben.

In den Fokusgruppen wurden im Sinne der klassischen Semantik sowohl ein semasiologischer als auch ein onomasiologischer Zugang gewählt. Im semasiologischen Zugang wird über die Bedeutung einzelner Geschmacksadjektive diskutiert, im onomasiologischen Zugang wird Geschmack aufgrund einer konkreten Degustation von Lebensmitteln und Getränken beschrieben. ${ }^{7}$

7 Es wurde festgestellt, dass sich, wenn beide Zugänge in der gleichen Fokusgruppe getestet wer- 
Die Auswahl der in den Fokusgruppen diskutierten Geschmackswörter basierte auf den im Abschnitt 2 beschriebenen Erhebungsverfahren und insbesondere auf den Ergebnissen des „Basic Taste Term Tests“; die Degustation von Lebensmitteln erfolgte nach intensiver Beratung durch LebensmittelsensorikerInnen der ETH Zürich. Bei der Datenerhebung und -aufbereitung haben wir uns an den Konventionen der linguistischen Gesprächsforschung orientiert. Die Fokusgruppendiskussionen wurden audiovisuell aufgezeichnet, da auch die nonverbale Kommunikation für die Erfassung der Semantik von Geschmackswörtern relevant ist. Die Diskussionen werden nach GAT (vgl. Selting et al. 1998) auf der Basis von Exmaralda (vgl. Schmidt/Wörner 2005) transkribiert.

\subsection{1 Überlegungen zur Methodenwahl}

Das Datenmaterial aus Fokusgruppendiskussionen soll es ermöglichen, das gesamte Wissen der Sprecherinnen und Sprecher in Bezug auf Geschmackswörter zu erfassen. Für die Methodenwahl gilt es deshalb, mehrere Faktoren zu berücksichtigen, damit ein kontrollierter Zugang zu ,inhaltlicheren“ Aspekten natürlicher Alltagssprache gewährleistet ist.

Geschmackswahrnehmungen sind individuell und subjektiv (vgl. Abschnitt 1), sie können erst im Gespräch interpersonell zugänglich gemacht werden. Standardisierte Erhebungsverfahren - wie Erhebungen durch Einzelinterviews oder Fragebögen - können daher eher weniger zum Erfolg führen, sondern ,man muss die Subjekte selbst zur Sprache kommen lassen; sie selbst sind zunächst die Experten für ihre eigenen Bedeutungsinhalte" (Mayring 2002:66).

Für die Untersuchung der Semantik von Geschmackswörtern bedarf es daher einer Methode, welche Untersuchungen darüber ermöglicht, wie sich das Individuum in der Gruppe auseinandersetzt und verständigt. Zentrale Stichworte dafür sind ,dialogische Spannung“ und „Reziprozität“ (vgl. auch Markova 2007:21): Die Interpretation eines Wortes kann sich im Gespräch ändern, und Geschmackswörter spezifizieren sich wechselseitig (vgl. Deppermann 2008:307). Einstellungen, Meinungen und Verhaltensweisen können zudem nicht in einer Isoliertheit erfasst werden, in der sie kaum je vorkommen.

In methodenpraktischer Hinsicht muss zudem auch das interpretative Aushandeln von Bedeutung berücksichtigt werden: Niessen (1977) fokussierte in seiner Konzeption der Gruppendiskussion auf die situationsabhängige Gruppenmeinung, die in jeder Handlungssituation neu ausgehandelt wird. Dieser Fokus ist wichtig, da die zentrale Frage ist, wie Gesprächsteilnehmer einander systematisch verdeutlichen, auf welche Weise die von ihnen verwendeten Geschmackswörter lokal zu verstehen sind. ${ }^{8}$ Nachfolgend wird erläutert, warum Fokusgruppen geeignet sind, diesen methodischen Überlegungen Rechnung zu tragen.

den, divergierende Ergebnisse zeigen. Hier zeigt sich die soziale und kulturelle (und das heißt auch: historische) Prägung nicht nur des Geschmackswortschatzes, sondern auch des kollektiven Wissens über Geschmack. Was dabei dann für die persönlichen Präferenzen bei der Wahl eines Produkts ausschlaggebend ist - das individuelle Geschmackserlebnis oder das kulturelle Wissen -, ist eine der weiterführenden Fragen, die sich hier zwangsläufig ergeben.

8 Deppermann $(2002,2007,2008)$ zeigt, dass für die Analyse von Gesprächen nicht von der Existenz kontextfrei gültiger Wortbedeutungen ausgegangen werden kann. In der Interaktion entsteht der Sinn einer Äußerung maßgeblich durch die Interpretation der Gesprächspartner, womit auch der Begriff der „Indexikalität““ (vgl. Garfinkel 1967:11) bedeutsam wird: Der Sinn einer sprachlichen Handlung wird aus dem subjektiven Bewusstsein des Handelnden in die objektive Interaktion verlagert. 


\subsubsection{Die Fokusgruppe als conceptual society}

Grundgedanke der Fokusgruppen ist, dass subjektive Bedeutungsstrukturen so stark in soziale Zusammenhänge eingebunden sind, dass sie nur in Gruppendiskussionen erhebbar sind (vgl. Mayring 2002:97). Darüber hinaus können durch ein homogenes Gruppensetting kollektive Orientierungen erfasst werden, die auch außerhalb der Diskussionssituation durch gemeinsame Erfahrungshorizonte bestehen (vgl. Lamnek 2005:68). Die beteiligten Sprecherinnen und Sprecher legen Einstellungen offen, die auch im Alltag ihr Fühlen und Handeln bestimmen. Mayring (vgl. Mayring 2002:97) weist darauf hin, dass solche subjektiven Bedeutungsstrukturen im Wesentlichen in sozialen Situationen, d.h. in Alltagssituationen entstehen. Fokusgruppen entsprechen in Gestaltung und Ablauf weitgehend „,natürlichen, alltäglichen Kommunikationssituationen“ (Lamnek 2005:52), d.h. es wird widersprochen, korrigiert, präzisiert, zusammengefasst usw. Daraus resultiert, dass das Datenmaterial reich an sprachlichen Handlungstypen, Emotionen und Geltungsweisen ist (vgl. Bohnsack et al. 2006:9).

Das Untersuchungsinteresse im Projekt SenS liegt in erster Linie auf inhaltlichen Phänomenen aus dem Bereich der Semantik, die interpretativ konstituiert sind. Daher ist es wichtig, einen empirischen Zugang zum sozial geteilten Geschmackswissen einer Kultur zu erhalten. Dies ist denn auch die große Stärke dieser Methode, wie eine Studie zum Dialog in Fokusgruppen von Marková et al. (2007:45) bestätigt:

[...] focus groups can generate rich verbal and interactional data and this is why we can say that they are potentially more social than other methods in social sciences. They allow the researcher to examine dynamic interactions that take place during communication as well as the formation, maintenance and change of socially shared knowledge.

Fokusgruppendiskussionen generieren Datenmaterial in zweifacher Hinsicht: Die Erhebungsmethode dient einerseits der Erfassung von Wortinhalten als „Instrument zur Rekonstruktion tiefer liegender semantischer Gehalte“ (Bohnsack et al. 2006:9). Das Datenmaterial aus Fokusgruppen ist jedoch nicht auf die Untersuchung inhaltlich-thematischer Aspekte limitiert, sondern auch Ausdrucksmuster und sprachliche Strategien der Bedeutungskonstitution können näher analysiert werden.

Die Fokusgruppe ist daher nicht mehr wie in der Sozialforschung nur als ,thinking society“, sondern als „talking society“ (Marková et al. 2007:46) zu verstehen. Bedeutungen werden in Fokusgruppen ausgehandelt und konstruiert; was letztlich entsteht, sind Begriffskonzepte. In Anlehnung an die Terminologie von Marková et al. (ibd.) und gerade hinsichtlich der Untersuchung zur Semantik des Geschmacks ist in Bezug auf Fokusgruppen eher von einer conceptual society zu sprechen. Die Fokusgruppe ermöglicht es, das gesamte bedeutungsrelevante Wissen in Bezug auf ein Wort bzw. in Bezug auf einen bestimmten Geschmack zu erschließen, da das verstehensrelevante Wissen rekonstruiert werden kann.

\subsubsection{Methodenfazit}

Die in der Linguistik bislang kaum erprobte Methode ${ }^{9}$ erweist sich für die Untersuchung der Semantik von Geschmackswörtern als besonders geeignet, da in einer quasi-natürlichen Gesprächssituation alltagsnahe Sprachdaten generiert werden.

9 Die Fokusgruppendiskussion als Methode der rekonstruktiv-qualitativen Sozialforschung hat das 
Die Sprachdaten aus den Fokusgruppendiskussionen belegen, wie in der Interaktion der Diskutierenden die Bedeutung von Geschmackswörtern dialogisch konstruiert und ausgehandelt wird. ${ }^{10}$ Da subjektive Geschmackserlebnisse auch sprachlich nicht direkt zugänglich gemacht werden können, treten die GesprächspartnerInnen regelrecht in Verhandlungen über ihre Sinneswahrnehmungen und über das Verständnis der jeweiligen Beschreibungen.

Die Methodenwahl berücksichtigt, dass es sich beim Geschmackswissen um soziales Wissen handelt, das in der dialogischen Kommunikation geteilt, geformt und gepflegt wird. Die Verwendung von Geschmackswörtern zielt nie allein auf die wahrnehmbare sensorische Qualität eines Lebensmittels, sondern es wird immer auch - zumindest implizit - Bezug genommen auf kulturelle Standards sowie auf kollektives Wissen, das zum Verstehen der Ausdrücke nötig ist.

Das folgende Kapitel soll anhand von Ausschnitten aus den Fokusgruppen illustrieren, welche Strategien zur Bedeutungspräzisierung bei der Verbalisierung von Geschmack von den Teilnehmenden verwendet werden.

\section{$4 \quad$ Strategien zur Versprachlichung von Geschmackswahrnehmungen}

Beim Reden über spezifische individuelle Geschmackswahrnehmungen wird selten direkt ein Geschmack benannt, sondern die Gesprächsteilnehmerinnen greifen auf verschiedene sprachliche Ausdrucksmöglichkeiten und verschiedene Strategien zur Umschreibung dieser Wahrnehmungen zurück.

Als Konsequenz für die Konzeption einer lexikografischen Datenbank, in welche auch systematisch mündliches Datenmaterial aufgenommen werden soll, heißt dies, dass die in den Gesprächen verwendeten Strategien mögliche Angabeklassen bilden könnten.

In den folgenden Unterkapiteln möchten wir exemplarisch anhand der Gespräche über die Geschmackswörter knackig, knusprig, fettig und über Brot darlegen, dass sich die Bedeutungskonstitution über verschiedene Abstraktions- und Ausführlichkeitsebenen ${ }^{11}$ erstrecken kann: von der Einzelwortnennung in Wortfeldern und in Kontrastierungen - den am häufigsten verwendeten Strategien - über die Nennung von Referenzen bis hin zu narrativen Sequenzen wie Handlungsszenarien und Bildern, den umfassendsten Verfahren zur Verbalisierung von Geschmack. Eine wichtige bedeutungskonstitutive Rolle spielt auch die Gestik, die sich oftmals gemeinsam mit metadiskursiven Bemerkungen zur Schwierigkeit der Verbalisierung zeigt und der sich das letzte Unterkapitel dieses Abschnitts widmet.

Potenzial, sich auch in der Linguistik als ernsthafte Alternative zu den standardisierten Erhebungsverfahren Einzelinterview und Fragebogen zu etablieren. Noch sind in der Sprachforschung wenige Anwendungen bekannt, an einer methodischen Auseinandersetzung fehlt es weitgehend. Im Bereich der linguistischen Forschung ist die Methode in den letzten Jahren vor allem von der „Wiener Schule“ der Kritischen Diskursanalyse angewendet worden (vgl. Wodak/Krzyzanowski 2008).

10 Diese Erkenntnis konnte semantiktheoretisch bislang nur unzureichend erklärt werden (vgl. Deppermann 2002, 2008).

11 Vgl. dazu auch Overlachs Studie zur „Sprache des Schmerzes“ (2008:45). 


\subsection{Strategien auf paradigmatischer Ebene}

\section{Diskussion von Wortfeldern}

Unser Datenmaterial zeigt, dass zu Beginn des Gesprächs die TeilnehmerInnen der Fokusgruppengespräche beim onomasiologischen Zugang um die Wahl des passenden Begriffs zur Beschreibung der Geschmackswahrnehmung bzw. beim semasiologischen Zugang um die Abgrenzung bereits genannter Geschmackswörter $\mathrm{zu}$ anderen bemüht sind. In beiden Formen von Fokusgruppengesprächen werden dabei Geschmackswörter in Bezug gesetzt zu bedeutungsähnlichen Begriffen, die dann im weiteren Verlauf des Gesprächs von einander abgegrenzt werden.

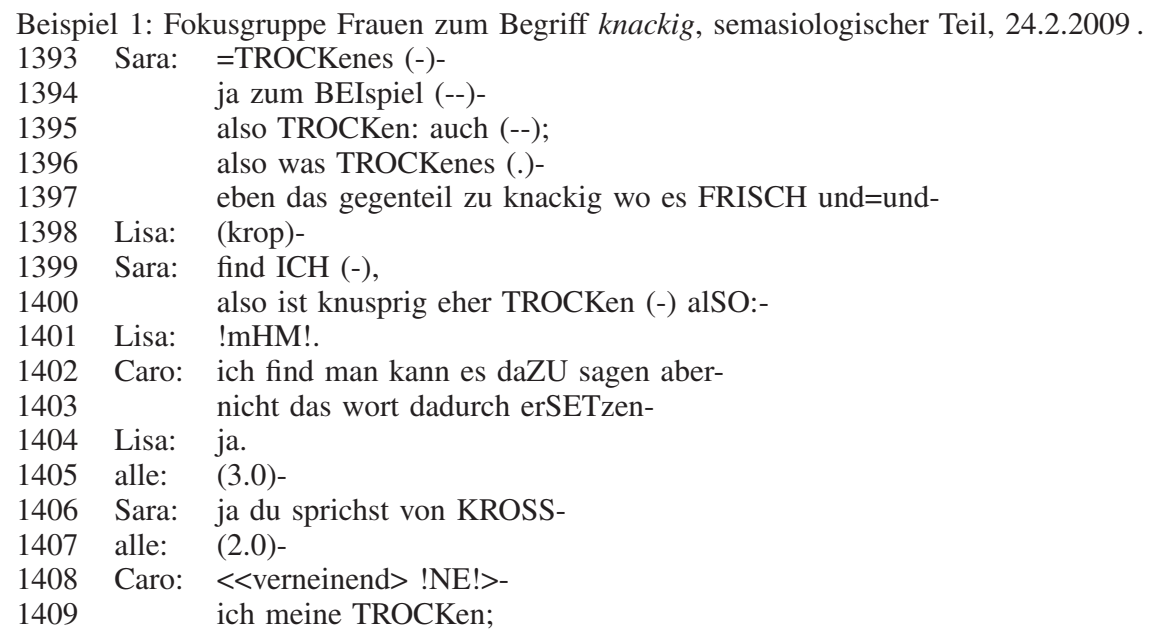

Der Ausschnitt aus der Frauen-Fokusgruppe zu knackig zeigt, dass die Teilnehmerin Sara zunächst versucht, die Bedeutung von trocken mit Hilfe der von ihr genannten Antonyme knackig und frisch näher zu beschreiben, schließlich wird von ihr trocken in Bezug gesetzt zu knusprig, um dann am Ende der Sequenz noch einmal von den Gesprächsteilnehmerinnen von kross abgesetzt zu werden.

\section{Kontrastierungen}

Eine ebenso häufig zu beobachtende Strategie wie die Abgrenzung von bedeutungsähnlichen Adjektiven in Wortfeldern ist die Kontrastierung. Bei der Kontrastierung wird versucht, über den Aufbau von Gegensätzen die Bedeutung eines Geschmackswortes zu spezifizieren. Die Kontrastierung kann einerseits als syntagmatische Beziehung (vgl. Deppermann 2008: 210ff.) realisiert sein, andererseits aber auch als paradigmatische. Letztere soll hier beschrieben werden.

Folgender Gesprächsausschnitt aus einer Männer-Fokusgruppe zum Wort fettig zeigt eine Kontrastierung zum Begriff ölig, die unter Rückgriff auf optische Eindrücke und Konsistenzmerkmale weiter ausdifferenziert wird:

Beispiel 2: Fokusgruppe Männer zum Begriff fettig, semasiologischer Teil, 24. 9. 2008.

1397 Peter: fettig ist irgendwie STUMpfer [glänzend-]

1398 Marc: [mhm-] 
1399 Peter: also so !MATT! glänzender irgendwie (-),

1400 ehm (-)-

1401 [während ölig] so !NETT! das licht reflektiert [irgendwie-]

1402 Marc: [das STIMMT-]

1403 Sven: [ja öl ist ] graZILer-

1404 [ja ÖLig'-]

1405 Peter: [und ölig GLÄNZTt;

1406 Anna: [((LACHT))] [ok ja,]

1407 Chris: [ja fett] ist FESter irgendwie

1408 Marc: also fett ist bei mir immer so der BLOCK (-),

1409 so IRgendwie was-

1410 ehm feste konsisTENZ,

1411 und ÖL ist halt-

1412 das FLIEsst halt (-);

1413

1414

1415

1416

1417

1418

1419

also das ist auch nicht so SCHMIEre,

Max: hm,

Marc: sondern schmiere ist auch noch relativ FETT,

eh FEST,

aber das ÖLige das (--)-

LÄUFT halt (-).

ALso das (2.0)-

1420

ist tatSÄCHlich ein unterschied. (--)

In der Fokusdiskussion der Frauengruppe zu fettig lässt sich ebenfalls eine Kontrastierung zum Wort fett beobachten, wie der folgende Transkriptionsausschnitt zeigt:

Beispiel 3: Fokusgruppe Frauen zum Begriff fettig, semasiologischer Teil, 1. 10. 2008.

Anna: hmman ist ja fett du kannst nicht sagen man ist FETTig; das GEHT ja nicht;

Jane: für mich wärs jetzt EHer so dass ähm (.)FETT tendenziell eher was ist (-)äh eher ein adjektiv is zur beschreibung von perSOnen auch wie du das gesagt hastaber wenn ICH ä:hm (-) ein lebensmittel als fett oder FETTig (.) beschreiben soll (kann ich) wie sagenist der unterschied dass fett noch viel stärker die !NE! gative konnotation hat also (.)FETT ähmdas ist wirklich ein lebensmittel wo definitiv !ZU! viel fett dran oder drin ist (-)und FETTig, das ist EHer so (- -) hm gefühl auf den FINgern, ähm (.) gefühl im !MUND!, find ichähm betrifft auch eher die: (.) die tatsächliche zuSAMMensetzung des nahrungsmittels; aber es ist nicht so STARK (--)äh (.) ABwertend (- -); wie FETTig (-); äh wie FETT (-); aber (-)vielLEICHT ist das auch (-)- 
354

355

356

357

358

359

360

361

ich WEISS nicht ob das objektiv so ist;

das wär jetzt EHer so (.)-

perSÖNlicher sprachgebrauch;

Anna: mhm,

alle: $\quad(-)$

Isa: aso dann ist fett NUR äh (.) negativ;

also weil FETTig hab-

Jane: ja-

Wie das Beispiel zeigt, wird der Begriff fett im Gegensatz zu fettig vorwiegend in Bezug auf Personen verwendet, enthält stärker negative Konnotationen und hat als Antonyme schlank, mager, dürr. In Bezug auf Lebensmittel wurden die Antonyme mager und fettarm genannt.

Die angeführten Beispiele von Verbalisierungsstrategien auf paradigmatischer Ebene zeigen durch ihre Unmittelbarkeit deutlich - vielleicht sogar eindrücklicher als dies in schriftsprachlichen Belegen möglich wäre - die Kontextabhängigkeit der diskutierten Begriffe, aber auch die Unsicherheiten bei der genauen Beschreibung sensorischer Wahrnehmungen im Alltag.

Durch die Berücksichtigung dieser Gesprächsbeispiele bei der Bedeutungsbeschreibung von Geschmackswörtern können wir auch zeigen, wie assoziativ (etwa in Zeile 1400, ,also ist knusprig eher TROCKen“) und wie individuell (etwa in Zeile 355/356 „,das wär jetzt EHer so (.)- perSÖNlicher sprachgebrauch“) das lexikalische Verständnis sein kann.

\subsection{Anbindung an die Lebenswelt}

Die Bedeutung von Geschmackswörtern wird - auch dies zeigt sich in unseren Daten sehr deutlich - in großem Maße durch anschauliche lebensweltliche Bezüge konkretisiert. Zentrale Verbalisierungsstrategien sind dabei die Nennung von prototypischen Produkten, der Bezug auf konkrete oder fiktive Handlungsszenarien bzw. die Konkretisierung mit Hilfe von Bildern, Szenen oder Beschreibungen aus der Werbung.

\section{Nennung prototypischer Referenzen}

Die Strategie der Bedeutungspräzisierung eines Geschmackswortes durch die Nennung prototypischer Referenzen wird in unseren Daten vorwiegend zu Beginn der Fokusgruppendiskussionen angewendet.

Wird kein Produkt zur Verkostung vorgelegt, sondern nur über eine Geschmacksempfindung gesprochen - sozusagen , aus der Erfahrung' bzw. aus dem entsprechenden Wissen heraus - , beginnen die Sprecherinnen und Sprecher häufig mit der Nennung prototypischer Nahrungsmittel, die sie dann weiter präzisierend beschreiben. Folgender Gesprächsausschnitt illustriert diese Strategie. Die vorangehende Frage der Moderatorin lautete: „Was fällt Ihnen zum Wort knackig spontan ein?“

Beispiel 4: Fokusgruppe Frauen zum Begriff knackig, semasiologischer Teil, 24.2.2009.

038 Sara: ja also bei mir ist es ÄHNlich,

039 ich hab auch-

$040 \quad$ äh=als erstes is mir FRISCH eingefallen;

$041 \quad$ FRIsche (--),

042 ähm=mir sind (-) WÜRSTchen eingefallen, 


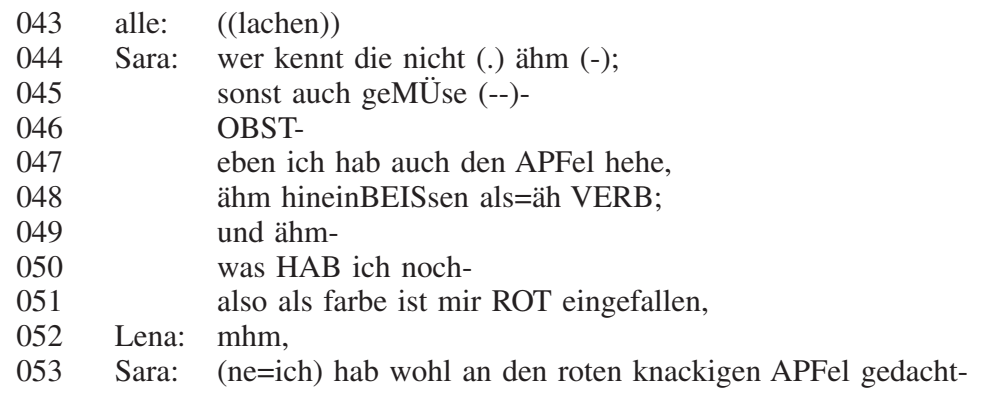

Die assoziative Bedeutungsstruktur der Geschmackswörter - wie hier im Beispiel zu knackig - kann auch im weiteren Verlauf des Gesprächs durch die Nennung von Referenzprodukten geprägt sein. Diese Einordnungen scheinen allgemein wichtig für die Bedeutungskonstitution zu sein. Auch bei der Degustation eines Produkts im onomasiologischen Teil der Fokusgruppengespräche wird die Frage, um welches Produkt es sich handelt und welchen anderen Produkten es geschmacklich ähnlich ist, so schnell wie möglich geklärt.

\section{Handlungsszenarien}

Im Verlaufe eines Gesprächs wird zur Bedeutungspräzisierung auch mit konkreten Situationsbezügen argumentiert, oder es werden mögliche und tatsächliche Erfahrungen mit verschiedenen Produkten geschildert. Dafür werden Handlungsszenarien entworfen.

Die folgenden Ausschnitte aus dem Fokusgruppengespräch zu knackig zeigen, wie die Fokusgruppenteilnehmer Bieg- und Brechbewegungen, hier insbesondere das Brechen einer Karotte oder das Hineinbeißen in einen Apfel als Handlungsszenarien nennen. Die Gestik hat dabei eine unterstützende Funktion.

Beispiel 5: Fokusgruppe Männer zum Begriff knackig, semasiologischer Teil, 24.2.2009.

261 Raul: [wie:, ]

262 die KArotte dass man die erst mal ein-

$263<<$ macht mit den Händen eine Brechbewegung $>-$

264 [bisschen>-]

265 Lena: [mhm- ]

266 Raul: und dann !KNA!ckt die eben durch-

267 und auch nur wenn sie frIsch ist dann gibts ja einen (-)-

268 Lena: mhm,

269 Raul: im gegensatz zur baNAne halt die dann wirklich nur biegt (.),

$270 \quad$ oder dann ganz leise bloss knackt die eben nicht FEst ist,

271 [in dem sinne also(.)-]

272 Lena: [mhm-]

273 Marc: [nickt ]

Beispiel 6: Fokusgruppe Männer zum Begriff knackig, semasiologischer Teil, 24.2.2009.

347 Raul: also das muss irgendwie -

348 wenn ich in den-

$349<<$ stellt mit Händen und Mund einen Apfelbiss dar >Apfel beisse>,

350 nicht nur dass der an sich knAckt,

351 sondern ich hab auch dieses -

352 [gerÄUsch dann im kopf -]

353 Marc: [((nickt)) ]

354 Dean: [mhm $(($ nickt $)) . \quad]$ 


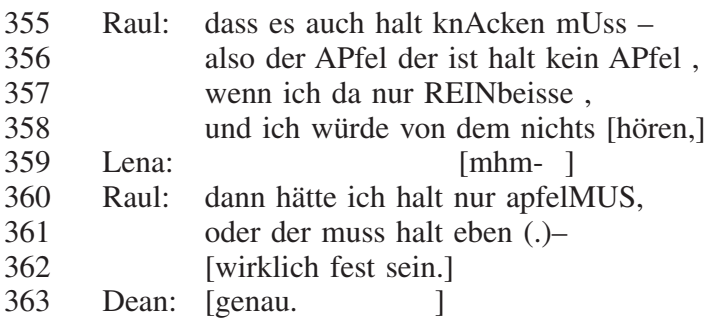

Wie die Passagen zeigen, spielt die Gestik in Bezug auf das Wort knackig eine bedeutungskonstitutive Rolle. Die Sprecherinnen können mit der Unterstützung von nonverbalen Strategien (Z. 263 und Z. 349) konkret erlebte Situationen verbalisieren, in denen sie knackig im Kontext eines Produktes ,erfahren' haben.

\section{Bilder und Szenen}

Geschmack kann man nicht teilen; Geschmackserlebnisse können nur über die Sprache ausgetauscht werden. Die Gesprächspartner sind darum bemüht, ihre Wahrnehmungen möglichst plastisch und nachempfindbar zu formulieren, um zu einem gegenseitigen Verstehen zu kommen.

Gerade bei der Verbalisierung von Geschmack werden immer auch Bilder und Szenen genannt: So beschreiben Fokusgruppenteilnehmer im Gespräch über knackig etwa eine Szene, in welcher Salat gewaschen wird, zudem werden Bilder von Äpfeln oder Salat mit Wassertropfen darauf als Zeichen der Frische genannt. Ebenso werden von den Teilnehmern auch Bilder aus der Produkt-Werbung genannt.

Beispiel 7: Fokusgruppe Männer zum Begriff knackig, semasiologischer Teil, 24.2.2009.

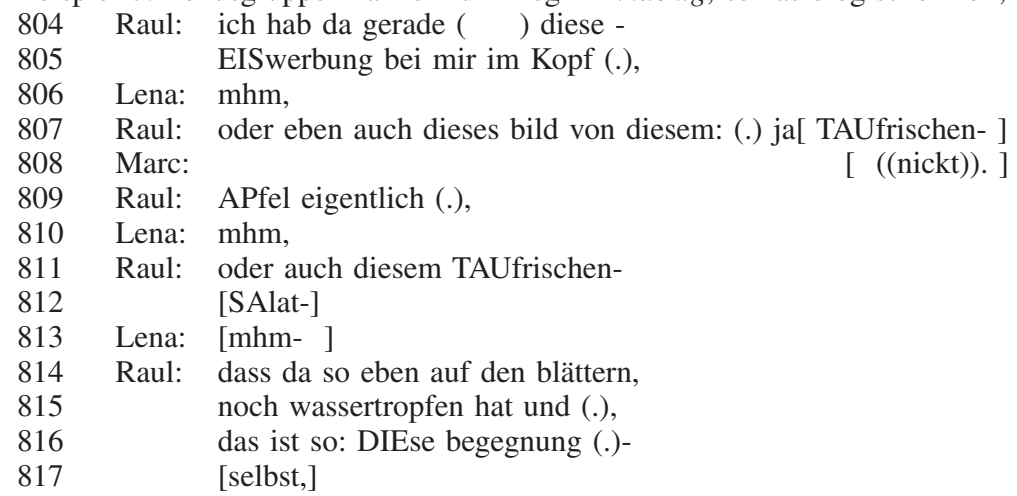

Beispiel 8: Fokusgruppe Frauen zum Begriff knackig, semasiologischer Teil, 24.2.2009.

221 Lena: saLAT ja,

222 gibts WEItere so (---).

223 bildliche assoziaTIONen oder zusammenhänge;

224 Lisa: <<macht schüttelnde Hin- und Herbewegung>geMÜse> gewaschen wird in der werbung,

225 so mit $m<<$ macht schüttelnde Hin- und Herbewegung $>$ SIEB unterm wasserhahn $>$;

226 Caro: oder wie $<<$ deutet auf Tina $>$ sie $>$ gesagt hat die KEKse die man so durchbricht. 
227 die dann so (--) KNACK machen.

228 Lena: [mhm oKE (---) ja-]

Beispiel 9: Fokusgruppe Frauen zum Produkt Brot, onomasiologischer Teil, 5.11.2009.

1046 Ida: also ich habs jetzt nochmals proBIERT-

1047 und ich hab die GANze zeit vorher versucht-

1048 nach irgendetwas zu SUchen;

1049 aber wie das (.) um das BROT-

1050 irgendwie den geSCHMACK zu beschreiben;

1051 und dann sind mir so (-)-

1052 eben so BAUMpilze in den sinn gekommen;

1053 also wenn du in den WALD gehst-

1054 Ria: [((hustet)) ]

1055 Ida: [und dann- ]

$1056<<$ mit den Händen Pilze anzeigend > überall-

1057

1058

1059

1060

1061 Ida: also (-) vielleicht auch ein bisschen so-

1062 wie TRÜffel oder so;

1063 aber irgendwas das aus dem BOden kommt;

1064 und aus dem wald-

1065 Joy: ERdig;

1066 Ida: [mhm (.) also ähm GANZ genau; ]

1067 Ada: [ERdig ist gut; ]

Wie in Beispiel 9 Bilder von Pilzen im Wald evoziert werden, um den Geruch bzw. den Geschmack von Brot zu präzisieren, so wird im Gespräch über fettig das Bild einer Tropfbewegung immer wieder aufgenommen.

Beispiele 10: Fokusgruppe Frauen zum Begriff fettig, semasiologischer Teil, 1.10.2008.

023 Jane: aber wenn man die dann so zuSAMMendrückt-

$024 \quad$ [dann] tropft das [FETT raus] [((lacht))]

025 Rosi: [dann>-]

026 [das ist ja dann-]

$027 \quad$ AUCH wieder dann tropf tropf-

$\mathrm{Zu}$ szenischen oder bildlichen Illustrationen wird also immer dann gegriffen, wenn es den Sprechern und Sprecherinnen nicht gelingt, ihre Vorstellungen in Bezug auf ein Geschmackswort lexikalisch zu präzisieren. Dabei spielt oft auch prozedurales Wissen eine wichtige Rolle. So wird etwa zur semantischen Präzisierung der Bedeutung von cremig zur Beschreibung eines Zubereitungsprozesses gegriffen, indem das Rühren in einem Topf geschildert wird.

\section{Gestik}

Die nonverbale Kommunikation spielt bei der Verbalisierung von Geschmackswahrnehmungen eine bedeutende Rolle, wie bereits aus den Beispielen 6 und 7 ersichtlich ist. Die Gestik tritt beim Reden über Geschmack als redebegleitende Kommunikation auf, vor allem dann, wenn Produkte degustiert werden. Gestik kann als Teil einer Äußerung andererseits auch verbale Handlungen ersetzen.

Das Geschmackswort fettig bspw. wurde mit den Begriffen triefend, nass, flüssig assoziiert. In den untersuchten Gesprächen wurde mehrere Male mit den Händen eine Tropf- 
bewegung dargestellt - so auch im Beispiel 10 - und gleichzeitig mit der Stimme lautmalerisch ein „tropf, tropf“ inszeniert. Hier tritt die Gestik redebegleitend als Unterstützung der verbalen Handlung auf:

Beispiele 11: Fokusgruppe Frauen zum Begriff fettig, semasiologischer Teil, 1.10.2008.

155 Rosi: ja ich denk an so an so getrocknete toMAten;

156 eingelegt in öl oder so-

157 die machen auch so-

$158<<$ macht mit der Hand eine Tropfbewegung >troptrop [tropf $>$ ]

Diese multimodalen Strategien helfen dem Sprecher oder der Sprecherin, Wörter, Gedanken, Bilder und Ideen zu aktivieren (vgl. Burgoon et al. 1996: 169). Sie sind gleichzeitig aber auch Indikatoren für die Schwierigkeit des Beschreibens von Geschmackswahrnehmungen. Das ist vor allem dann der Fall, wenn Gesten die verbalen Handlungen gänzlich ersetzen, wie es im folgenden Beispiel ersichtlich ist. Hier ersetzt die Geste des Karottenbrechens das Wort brechen:

Beispiel 12: Fokusgruppe Männer zum Begriff knackig, semasiologischer Teil, 24.2.2009.

261 Raul: [wie:,]

262 die KArotte dass man die erst mal ein-

$263<<$ macht mit den Händen eine Brechbewegung > -

264 [bisschen>-]

265 Lena: [mhm- ]

266 Raul: und dann !KNA!ckt die eben durch-

267 und auch nur wenn sie frIsch ist dann gibts ja einen (-)-

Diese Schwierigkeit bei der Verbalisierung von Geschmackswahrnehmungen zeigt sich verstärkt in Situationen, wenn Gesten in Verbindung mit einem Metadiskurs wie „Das ist ziemlich schwer zu erklären.“ (Bsp.14, Z.1636) auftreten. Diese Kombination findet sich im folgenden Transkriptionsausschnitt. Hier versucht die Teilnehmerin Ida zu beschreiben, wie das eben degustierte Brot schmeckt. Dabei hält sie sich die Finger an die Kehle, um zu verorten, wo es im Hals brennt.

Beispiel 13: Fokusgruppe Frauen zum Produkt Brot, onomasiologischer Teil, 5.11.2009.

1620 Ida: vielleicht rauch (.) dass es im RAUCH war;

1621 Ria: [((hustet)) ]

1622 Ida: [und brandig einfach (.) dass ein FEUer daneben war; ]

1623 Ada: ah dann wär glaub ich das DREI-

1624 das wär Eher son rauch (.) son RAUCHbrot wie das eins;

1625 Pia: also ich hab (.) einfach bei dem bei dem RAUchig-

1626 das mehr auf die KRUSte bezogen;

1627 da fand ich einfach eben-

1628

1629

1630

1631

1632

1633

1634

1635

1636

1637

dass das an dieses FEUer (.) erinnert hat;

und bei BRANdig (.) hab ich immer so ein bisschen das gefühl-

es könnt auch (-) son bisschen BEIzen;

also (.) wenn man HOCH konzentrierten ALkohol hat dann-

$<<$ Finger an die Kehle haltend $>$ bekommt den dann hier in-

in so diesen HALS: $>$

[HALSgebiete eigentlich- ]

alle: [((lachen) $)]$

Pia: das ist ZIEMlich schwer zu erKLÄren;

ähm dann hab ich manchmal das geFÜHL- 
1638 Joy: [brennt << $\quad$ grinsend $>\mathrm{hm} \mathrm{hm>;}]$

1639 Pia: [ja es br es BRENNT auch wenn sachen Überdosiert sind- ]

1640 Ria: $\quad[\mathrm{mhm}-$ ]

Die angeführten Beispiele zeigen, dass sich Sprecherinnen und Sprecher bei der Verbalisierung von individuellen Wahrnehmungen vielfältiger Strategien sowohl sprachlicher als auch multimodaler Art bedienen. Gerade deren Häufigkeit und deren systematische Verwendung in den untersuchten Fokusgruppengesprächen führen zu dem Schluss, dass diese bei der Bedeutungsbeschreibung von Geschmackswörtern unbedingt berücksichtigt werden müssen.

\section{Fazit}

Die Bedeutungsbeschreibung von Geschmacksadjektiven stellt uns vor besondere Herausforderungen - sowohl bei der Erhebung von Sprachmaterial als auch bei der Art und Weise, wie diese Wörter lexikografisch beschrieben werden müssen.

Geschmacksadjektive sind meistens polysem und werden auch außerhalb von Geschmackskontexten vielfach - häufig auch metaphorisch - verwendet. Für die Untersuchung von Geschmacksadjektiven stehen wir deshalb vor der Herausforderung, Datenmaterial zu erheben, in dem das Wort ausschließlich für die Beschreibung von Geschmackswahrnehmungen verwendet wird. Bei der Auswertung schriftsprachlicher Korpora können wir dies sicherstellen, indem wir nach Belegen suchen, in denen durch die Verwendung der Lexeme Geschmack, schmecken, Aroma der Verwendungskontext vorgegeben ist.

Für die Erhebung von Daten gesprochener Sprache haben sich dagegen Fokusgruppendiskussionen als methodisch besonders geeignet erwiesen. Folgende Punkte können wir als vorläufige Ergebnisse unserer Untersuchung von Fokusgruppengesprächen über Geschmackswahrnehmungen und deren Verbalisierung festhalten:

- Grundsätzlich ist festzuhalten, dass die Semantik von Wörtern bisher kaum Untersuchungsgegenstand von Gesprächsanalysen ist (vgl. Deppermann 2008: 217f). Die in den Fokusgruppen beobachteten Formulierungsstrategien zeigen aber, dass es durchaus Sinn macht und auch notwendig ist, die Bedeutung von Wörtern im Gespräch zu untersuchen. Die Bedeutung von Geschmackswörtern wird in der Gruppeninteraktion ausgehandelt und hergestellt. Die Frage nach der Bedeutung von Geschmackswörtern erfordert also in besonders hohem Maß auch die Berücksichtigung pragmatischer Faktoren. Die Forschungsperspektive der kontextuellen Bedeutungskonstitution erlaubt keine Trennung von Semantik und Pragmatik (siehe auch Deppermann 2002:27).

- Die Methodik der Fokusgruppengespräche ermöglicht die Beantwortung einer weiteren Forschungsfrage, die es zu berücksichtigen gilt: Wie ist das semantische Wissen von Sprechern und Sprecherinnen des Deutschen mit Bezug auf Geschmackswahrnehmungen konstruiert?

Wir stellten bereits fest, dass semantisches Wissen auch bei Geschmackswörtern prozedurales Wissen umfasst, obwohl man dies zunächst eher nicht vermuten würde. Dass „Konzertbesuch“ prozedurales Wissen abruft, leuchtet auf den ersten Blick ein. Dass aber beispielsweise ein Geschmackswort wie cremig das auch tut - den Prozess der 
Herstellung einer cremigen Substanz wie das Schlagen von Schlagsahne oder das Rühren einer festeren Masse zu einer Creme -, das ist weniger zu vermuten, zeigt sich aber deutlich aus den Fokusgruppengesprächen.

- Die Frage nach dem Verhältnis von individueller Wahrnehmung und der Möglichkeit der interpersonellen Kommunikation ist eine der Grundfragen der Semantik. Feststeht: Die Geschmacksempfindungen anderer sind uns nicht zugänglich, man kann sie nicht teilen - was wir teilen können, sind nur Worte. Geschmack ist darum auch eine zutiefst sprachliche Erfahrung.

- Ausgehend von einer Theorie des sprachlichen Handelns (Auer 1999) wird die sprachliche Äußerung nicht nur als Ausdruck, sondern als Konstituens des Geschmacks betrachtet. Damit sind wir vordergründig bei der zentralen Frage, in welcher Weise Sprache als Medium (Günthner/Linke 2006) nicht nur die Kommunizierbarkeit von Sinneseindrücken, sondern auch die Geschmackswahrnehmung selbst beeinflusst. Die Forschungsergebnisse sollen auch Rückschlüsse auf den Zusammenhang zwischen dem sprachlichen Ausdruck und der Wahrnehmung des Geschmacks ermöglichen.

Was für Konsequenzen haben die bisherigen Ergebnisse - aber auch die daraus entstandenen weiterführenden Forschungsfragen - für die Konzeption einer lexikografischen Datenbank?

Der Individualität von Geschmackswahrnehmungen und der von den sprachlichen Fähigkeiten der einzelnen Sprecherinnen und Sprecher abhängigen Verbalisierungsstrategien müssen in einer Datenbank, in welcher der Geschmackswortschatz des Deutschen beschrieben werden soll, Rechnung getragen werden. Es hat sich gezeigt, dass die in Abschnitt 4 beschriebenen Strategien - die Diskussion eines Geschmacksworts innerhalb eines Wortfelds, dessen Kontrastierungen zu anderen Geschmackswörtern, die Nennungen prototypischer Referenzen, die Schilderungen von Handlungsszenarien und Bildern bzw. Szenen sowie der Einsatz von Gestik - in den Fokusgruppendiskussionen immer wieder zu finden sind.

Es würde sich daher anbieten, bei der Konzeption der Datenbank - neben den klassischen Angaben, die auf der Auswertung schriftsprachlicher Belege beruhen - diesen Verbalisierungsstrategien zu folgen und sie als Angabeklassen für die lexikografische Beschreibung zu wählen. Mit diesem Vorgehen könnten wir systematisch Datenmaterial aus Gesprächen für die lexikografische Beschreibung eines Wortschatzausschnitts nutzen. Ergänzt werden müssten die entsprechenden Angabeklassen ggf. um Metakommentare, welche den Kontext des Gesprächsausschnitts oder sonstige Angaben zum Gespräch enthalten.

Die Untersuchung der Fokusgruppengespräche hat auch gezeigt, dass das mündliche Datenmaterial äußerst interessante Hinweise auf die komplexe Semantik von Geschmackswörtern liefert. So scheint prozedurales Wissen für bestimmte Geschmackswörter von besonderer Bedeutung zu sein (etwa cremig), bei anderen Geschmackswörtern sind es eher Bilder und Szenen (etwa bei knackig), die unmittelbar bei den GesprächsteilnehmerInnen evoziert werden. Solche Aspekte semantischen Wissens unterscheiden sich deutlich von den eher auf der Darstellung überindividueller Aspekte beruhenden Bedeutungsbeschreibungen aktueller Wörterbücher - Beispielangaben in Wörterbüchern dienen hier nicht als Nachweis, sondern als Illustration der Bedeutungsbeschreibung. Eine andere Funktion hätten dagegen die Ausschnitte aus Transkripten oder Audio- bzw. Videodokumenten, in welcher die Formulierungsstrategien präsentiert werden könnten. Die entsprechenden Daten führen dem Benutzer zum einen die Individualität der Aussagen vor Augen, geben ihm zum anderen 
aber auch die Möglichkeit, den entsprechenden Ausschnitt selbst zu interpretieren. Damit wäre das lexikografische Vorgehen bei der Einbindung von Gesprächssausschnitten so transparent wie möglich. Unumgänglich ist in diesem Zusammenhang die genaue Auszeichnung der Quellengrundlage bei den jeweiligen Angaben.

Mit der systematischen Einbindung von mündlichem Sprachmaterial in eine lexikografische Datenbank werden aber auch die Mehrwerte, welcher der Wechsel von gedruckten lexikografischen Werken zu digitalen Datenbanken bedeutet, genutzt; hierbei bedarf es jedoch nach wie vor einer lexikografischen Methodik (vgl. Haß-Zumkehr 2001:250).

\section{Literatur}

Abel 2009 = Abel, Andrea / Stuflesser, Mathias: Language-Bridges- Interviewstudie zum Zusammenspiel von Überzeugungen, Erfahrungen und Sprachenlernen: Methodenfragen und Ergebnisauswertung. In: Werlen, Erika / Tissot, Fabienne (Hgg.): Sprachvermittlung in Europa. Beiträge der Angewanden Linguistik zum Dialog zwischen Wissenschaft und Gesellschaft. Hohengehren: Schneider, 2009, S. 75-87.

Auer 1999 = Auer, Peter: Sprachliche Interaktion. Eine Einführung anhand von 22 Klassikern. Konzepte der Sprach- und Literaturwissenschaft. Bd. 60. Tübingen: Niemeyer, 1999.

Bieler et al. $($ im Druck) = Bieler, Larissa Margot / Wagner, Daniela / Ziem, Alexander (im Druck): Zur Versprachlichung von Geschmackseindrücken. Erscheint in: Proceedings zu den 5. Tagen der Schweizer Linguistik.

Birbaumer/Schmidt 2006 = Birbaumer, Niels / Schmidt, Robert F.: Biologische Psychologie. 6., vollständig überarbeitete und ergänzte Auflage. Berlin/Heidelberg: Springer (Springer-Lehrbuch), 2006.

Bohnsack et al. 2006 = Bohnsack, Ralf et al.: Einleitung. Gruppendiskussionen als Methode rekonstruktiver Sozialforschung. In: Bohnsack, Ralf et al. (Hgg.): Das Gruppendiskussionsverfahren in der Forschungspraxis. Opladen: Budrich, 2006, S.7-22.

Burgoon et al. $1996=$ Burgoon, Judee K. et al.: Nonverbal Communication. The Unspoken Dialogue. Second Edition. New York et al.: McGraw-Hill, 1996.

Deppermann 2000 = Deppermann, Arnulf: „Ethnographische Gesprächsanalyse: Zu Nutzen und Notwendigkeit von Ethnographie für die Konversationsanalyse“. In: Gesprächsforschung Online-Zeitschrift zur verbalen Interaktion, 1/2000. S. 96-124. (www.gesprächsforschung-ozs.de).

Deppermann 2002 = Deppermann, Arnulf: „Von der Kognition zur verbalen Interaktion“. In: Deppermann, Arnulf / Spranz-Fogasy, Thomas (Hgg.) (2002): „be-deuten: Wie Bedeutung im Gespräch entsteht“. Tübingen: Stauffenburg, S. 7-33. (Stauffenburg Linguistik 27).

Deppermann 2007 = Deppermann, Arnulf: „Grammatik und Semantik aus gesprächsanalytischer Sicht“. In: Günthner, Susanne et al. (Hgg.): Linguistik - Impulse und Tendenzen. Bd. 14. Berlin/ New York: de Gruyter, 2007.

Deppermann 2008 = Deppermann, Arnulf: Verstehen im Gespräch. In: Kämper, Heidrun / Eichinger, Ludwig M. (Hgg.): Sprache - Kognition - Kultur. Sprache zwischen mentaler Struktur und kultureller Prägung. S. 225-261. Berlin/New York: de Gruyter (= Jahrbücher des Instituts für deutsche Sprache 2007).

Günthner/Linke 2006 = Günthner, Susanne / Linke, Angelika: „Linguistik und Kulturanalyse. Ansichten eines symbiotischen Verhältnisses“. In: Ágel, Vilmos et al. (Hgg.): „Linguistik und Kulturalanalyse“. Zeitschrift für germanistische Linguistik, 34.1/2. Berlin: de Gruyter, 2006, S. 1-27.

Haß-Zumkehr 2001 = Ulrike Haß-Zumkehr: Deutsche Wörterbücher - Brennpunkt von Sprach- und Kulturgeschichte. Berlin 2001 (de-Gruyter-Studienbuch)

Lamnek 2005 = Lamnek, Siegfried: Gruppendiskussion. 2. Auflage. Weinheim/Basel: Beltz, 2005. 
Marková, Ivana et al. (2007): Dialogue in Fokus Groups. London/Oakille: Equinox.

Mayring 2002 = Mayring, Philipp: Einführung in die qualitative Sozialforschung. Weinheim: Psychologie Verlags Union, 2002.

Morgan/Corbett 1989 = Morgan, Gerry / Corbett, Greville: Russian colour term salience. In: RussianLinguistics. 13, 1989, S. 125-41.

Niessen 1977 = Niessen, Manfred: Gruppendiskussion. Interpretative Methodologie - Methodenbegründung - Anwendung. Fink: München, 1997.

Overlach 2008 = Overlach, Fabian: Sprache des Schmerzes - Sprechen über Schmerzen. Eine grammatisch-semantische und gesprächsanalytische Untersuchung von Schmerzausdrücken im Deutschen. Berlin/New York: de Gruyter, 2008.

Schäffer 2003 = Schäffer, Burkhard: Gruppendiskussion. In: Bohnsack, Ralf et al. (Hgg.): Hauptbegriffe Qualitative Sozialforschung. Opladen: Leske + Budrich, 2003, S. 75-80.

Schmidt/Wörner 2005 = Schmidt, Thomas /Wörner, Kai: Erstellen und Analysieren von Gesprächskorpora mit Exmaralda. Gesprächsforschung - Online-Zeitschrift zur verbalen Interaktion, Ausgabe 6 (2005), S. 171-195 (www.gespraechsforschung-ozs.de).

Selting et al. 1998 = Selting, Margret et al.: Gesprächsanalytisches Transkriptionssystem (GAT). In: Linguistische Berichte 173, 1998, S. 91-122.

Ternes et al.(Hgg) 2005 = Ternes, Waldemar / Täufel, Alfred / Tunger, Liselotte / Zobel, Martin (Hgg.) Lebensmittel-Lexikon. 2005. Hamburg: B. Behr's Verlag.

Wagner 2009 = Wagner, Daniela: Semantische Dimensionen von Geschmacksadjektiven. Eine Skizze zum Adjektiv würzig. In: Lavric, Eva / Konzett, Carmen (Hgg.): Food and Language. Sprache und Essen (Inntrans. Innsbrucker Beiträge zu Sprache, Kultur und Translation). Frankfurt/M. et al.: Peter Lang, 2009.

Wodak/ Krzyzanowski (Hgg.) 2008 = Wodak, Ruth / Krzyzanowski, Michal (Hgg.): Qualitative Discourse Analysis in the Social Sciences. New York: Palgrave Macmillan, 2008. 